Proceedings of the 2012 Winter Simulation Conference

F. Boulaire, M. Utting, R. Drogemuller, G. Ledwich, and I. Ziari

\title{
A HYBRID SIMULATION FRAMEWORK TO ASSESS THE IMPACT OF RENEWABLE GENERATORS ON A DISTRIBUTION NETWORK
}

\author{
Fanny Boulaire \\ Mark Utting \\ Robin Drogemuller \\ Queensland University of Technology \\ Faculty of Creative Industries \\ 126 Margaret Street - level 12 \\ Brisbane, Qld 4000, AUSTRALIA
}

\author{
Gerard Ledwich \\ Iman Ziari \\ Queensland University of Technology \\ Faculty of Science and Engineering \\ Gardens Point - S Block \\ Brisbane, Qld 4000, AUSTRALIA
}

\begin{abstract}
With an increasing number of small-scale renewable generator installations, distribution network planners are faced with new technical challenges (intermittent load flows, network imbalances...). Then again, these decentralized generators (DGs) present opportunities regarding savings on network infrastructure if installed at strategic locations. How can we consider both of these aspects when building decision tools for planning future distribution networks? This paper presents a simulation framework which combines two modeling techniques: agent-based modeling (ABM) and particle swarm optimization (PSO). ABM is used to represent the different system units of the network accurately and dynamically, simulating over short time-periods. PSO is then used to find the most economical configuration of DGs over longer periods of time. The infrastructure of the framework is introduced, presenting the two modeling techniques and their integration. A case study of Townsville, Australia, is then used to illustrate the platform implementation and the outputs of a simulation.
\end{abstract}

\section{INTRODUCTION}

Following commitments by Australia to reduce its greenhouse gas emissions, the target of generating $20 \%$ of energy using renewables by 2020 has been set (Australian Government 2011). To help reach this goal, measures have been put into place by the government to encourage individuals to invest in smallscale generation units, such as rooftop solar panels (Queensland Government 2011) and (Australian Government 2012). These decentralized generators (DGs) have advantages not only in terms of $\mathrm{CO}_{2}$ emission reductions, but they can also support the peak load growth if installed at strategic locations on the network, potentially saving money on infrastructure investments. Indeed, Australia's peak electricity consumption, which is the metric used when designing electricity networks (generation and infrastructure), has rapidly increased over the last few years. For example, Queensland has observed a 7\% increase in peak demand on average over the last 7 years (Queensland Government 2009) with up to 14\% increase for some years. Knowing that approximately $10 \%$ of the electricity distribution network capacity is built to meet the peak demand, which only occurs for approximately $1 \%$ of the time (Queensland Government 2009), understanding how DGs can best be used to shave peak demand is crucial when planning for infrastructure maintenance and extension.

However, despite these opportunities, DGs are causing new technical challenges to distribution companies, which have to manage intermittent load flows, network imbalances... preventing the installation of DGs in some areas that have reached a high percentage of penetration (Hall 2011). While some countries in Europe have managed to reach quite high percentages of variable renewable generation (over 10\% for wind for example), this was achieved thanks to cross-border transmission links (Kirby and Milligan 2008). Australia, being isolated from other countries, needs to deal with the technical challenges in a different manner. Also, because of the lack of experience with high penetration rates of variable electricity 
resources worldwide, very little is known about what will happen when these generation types increase to very large proportions (Komor 2009). Consequently, distribution companies are looking at understanding such effects using modeling techniques which can investigate different scenarios for planning future energy grids.

The project described in this paper aims at assessing the impact of the large-scale introduction of renewables on a distribution network, managed by Ergon Energy, one of the two electricity distribution companies that serve the state of Queensland, Australia. Due to the network spare nature (it covers 1.7 million square kilometers and provides power to approximately 680,000 homes and businesses across regional and rural Queensland) and its vast number of assets (approximately 150,000 kilometers of power lines, 1 millions of poles) many components need to be taken into account, in addition to the introduction of renewable generators, when planning for the future network. A question then arises: "How can we best manage the network that will allow for a mix of network extension and integration of distributed generation that will optimize the overall system in terms of cost benefits and demand?"

To answer this problem a framework has been developed. This framework supports the examination of the system as a whole, so that the impact of a change in one part of a system on other parts can be assessed. Contrary to the current practice where critical areas are identified and assessed using static analyses to assess the level of capacity required to service an area (Ergon Energy 2010), the framework considers the system as a whole, whether or not the areas have reached capacity. This means that all the components making up the network need to be represented and the evolution of the system needs to be captured over-time. As such, a dynamic analysis of the distribution system is required that can assess both the consumption (for end-user premises) and the production (of renewable DGs) over time and at specific locations. This dynamic assessment can then be used in the analysis to find the most economical configuration of DGs for long-term planning.

The framework presented in this paper consequently performs analyses over different time scales and calls on different techniques. Two modeling techniques are used: agent-based modeling (ABM) and particle swarm optimization (PSO). ABM is used to represent the different system units accurately and dynamically, following the changes over time and at different levels of detail in the distribution network. Load duration curves which are output from the ABM simulation are then used in the PSO module to find the most economical mix of network extension and integration of distributed generation over long periods of time. Combining these two modeling techniques allows taking advantage of each method's strength to obtain the necessary simulations over different timeframes. Another key feature of this framework is its ability to model both (1) the technical network constraints and (2) the economic and sustainability challenges of minimizing cost and carbon intensity.

This paper presents in the first instance the overall architecture of the framework, introducing the two modeling techniques used in the framework's components and their interactions. The implementation of the platform is then illustrated using a case study of Townsville, Australia and results of the discrete-event simulation are discussed.

\section{AGENT-BASED MODELING AND PARTICLE SWARM OPTIMIZATION}

Agent-based modeling is a technique used to model complex systems comprised of autonomous and interacting agents. An agent-based model (ABM) can be described as a "class of computational models for simulating the actions and interactions of autonomous agents (both individual and collective entities such as organizations or groups) with a view to assessing their effects on the system as a whole" (Wikipedia 2011). This modeling technique is becoming increasingly widespread with applications in domains as diversified as consumer behavior modeling, traffic congestion analysis and electricity market simulations. One of the reasons for such an uptake of this technique is that we live in an increasingly complex world, but also that the computational power currently available allows computing "large-scale micro-simulation models that would not have been plausible just a couple of years ago" (Macal and North 2006).

As its name indicates, agent-based models are composed of agents. While there is no universal agreement on the definition of an agent, most modelers share the same view regarding the properties of an 
agent. Agents have the following attributes (Macal and North 2005): agents are autonomous and selfdirected, they are modular or self-contained, and they are social, interacting with other agents. They can also have additional properties such that: they live in an environment, they have explicit goals that drive their behavior, and they learn and adapt their behaviors based on their experiences.

These agents can be specified at various scales, defining the granularity of the model. As they are autonomous, decision-making heuristics, learning rules or adaptive processes have to be included in the model as well as an interaction topology. Such properties make using ABM for the electricity network adequate. Indeed, the components making up the network can be defined as agents, with the network topology captured through the agents' interactions. The behavior of the agents can be defined independently reflecting the way that end-users consume electricity according to their needs and the activities they undertake. Through building the system from the lower level (e.g. where an agent can represent an asset on the network such as a transformer) to a higher level (e.g. a substation), an emergent phenomenon can be observed and the system can be assessed as a whole. Additionally, the encapsulation of algorithms and data within each agent improves flexibility when configuring alternative scenarios.

While agent's behaviors can be optimized, a clear distinction has to be made between agent-based modeling optimization and full optimization of the system, as it is fundamentally different in nature and purpose. The agents' intention is not to optimize the system as a whole; they typically try to optimize only their own behavior. This could be regarded as doing local optimization over the system, at the agent level, which might result in a very non-optimal system. While ABM is not an appropriate technique for system optimization, it can be combined with optimization methods to bring a compromise to the full analysis, as implemented in this framework.

Optimization was used as the technique to plan for the most economical mix of renewables to be installed on the distribution network. Because optimal planning of distribution networks is a large-scale mixed-integer and nonlinear problem, classified as an NP-hard problem, conventional optimization methods (e.g. linear programing, integer programing) are not good candidates for such problem. However, PSO, which is a population-based and self-adaptive optimization technique, is known to effectively solve these types of problems, and was employed here. PSO has been effectively applied to different optimization problems in power systems, one of which is the generation expansion problem which "consists in determining what, when, where and how to install new generation units in order to meet the power system requirements while constraints regarding load demand, reliability, operating conditions, power quality, and security are met" (del Valle, Venayagamoorthy et al. 2008). This problem is formulated so that the least cost of the investment as expressed in the objective function can be found. Such problem is usually applied for the placement of high-voltage generation units, but is adequate to the case of low-voltage generators.

PSO, which is a stochastic-based method, handles a population of individuals in parallel, to probe areas where the optimal solution is located. The individuals are called particles and the population is called a swarm. This optimization method leads particles in a swarm toward the optimal point using an adaptive velocity. Many different variants of the basic PSO can be used (del Valle, Venayagamoorthy et al. 2008); the one chosen for this problem is a hybrid of evolutionary programing and PSO, as it uses mutation and cross-over when updating the position of the particle, as described in (Ziari, Ledwich et al. 2010).

\section{RELATED WORK}

A large number of tools are available for various analyses in the electricity sector. For example, in their review (Foley, Ó Gallachóir et al. 2010) have described 7 models applied to electricity systems; in (Connolly, Lund et al. 2010) 68 tools have been identified for analyzing the integration of renewable into various energy systems, 37 of which are described in more detail; and many more tools are available that can be found on the internet, for example (Powertech Labs Inc 2012; ROAM Consulting 2012), without counting the ones developed internally by the different electricity authorities. In many of these tools, analyses of the feasibility in installing renewables at given locations, calculating how much they are expected to produce at different times of the year, whether it be small-scale or large-scale renewables, is 
possible. These types of tools aim at finding the most profitable location to install large-scale generators, and therefore detailed analyses are available. However, no transition in models from site assessment to state-wide assessment of impact of renewables was found, especially when it comes to linking the electricity generation from the renewable systems to the technical constraints of the distribution network.

A framework, MOSAIK (Schutte, Scherfke et al. 2012) is currently being developed that aims at linking existing simulation models automatically. For this, the technology regarding model interfacing and data flow management is being developed. While this work aims at putting existing models together, it does not address the problem of putting optimization together with models, especially when dealing with linking data at different levels of granularity and over different time periods. The work presented in this paper is innovative in the way that it links planning methods (through the PSO module) taking into account the variability in the network behavior (through the ABM module) which is usually only considered when performing analyses at the operational level. The data used for the optimization module is obtained after manipulation and aggregation of the data output by the ABM module and the optimization is done over longer time horizons than the ABM. Another framework (GridLAB-D) that aims at providing similar functions as the one proposed in this paper (Chassin, Schneider et al. 2008) is also currently being developed. The GridLAB-D framework though, only has a few of its planned modules currently implemented, and none deal with the optimization of the placement of DGs.

The work presented here offers an innovative way of combining models at different levels of granularity (different geographical and time scales) to optimize the investments of the distribution network. Network augmentation and introduction of DGs are considered in the optimization part of the simulation informed by the behavior of the different entities while in operation, allowing capturing the actual variability in the assets performance and not relying on averages only. Additionally, this framework models both (1) the technical network constraints and (2) the economic and sustainability challenges of minimizing cost and carbon intensity.

\section{MODULAR FRAMEWORK ARCHITECTURE}

For this project, not only does the simulation require the implementation of two modeling techniques but it also requires a multi-level representation. The simulation needs to happen over two time horizons (short-term management and long-term planning) and needs to be location specific (where load patterns evolve depending on the area of consumption and generation). Figure 1 gives an overview of the framework, showing how the different components represent the systems entities and their relationships, as well as the use of the two modeling techniques.

As can be seen, the framework has different levels of complexity; the simulation is done over varying levels of granularity in terms of agents and time. Agents in this context can be individual network assets, but they can also be represented as groups of agents whose characteristics result from the aggregation of finer-grain agents. As an example, an agent can be a single photovoltaic unit on a house at the finer level of granularity, or at a coarser level of granularity, it can represent the photovoltaic characteristics of a suburb in terms of penetration rate and generation capacity.

The time scale for this framework contains three levels of detail: $1 / 2$ hourly, yearly and five-yearly. Simulation runs are performed on a $1 / 2$-hourly basis for the calculation of the electricity demand at a specific location and for every day of the year; the calculation is such that the overall demand to the grid consists of the electricity required by a household, for example, minus the electricity generated by the photovoltaic unit, if installed, whose output depends on the solar availability. These $1 / 2$-hourly calculations are then aggregated and yearly simulations can be performed that consider growth in demand due to factors such as population growth or increase in PV penetration. These calculations are done in the ABM module, where the network and its characteristics are defined. The outputs of these calculations are manipulated, so that they can be used for the 5-yearly simulation, which corresponds to the time cycle used when developing network improvement plans. A set of load duration curves is used as an input to a load flow analysis which is iteratively used with the PSO program. Different configurations of the network are as- 
sessed and the output results in selecting one configuration for which costs (capital, maintenance and depreciation) of the network have been minimized under the network constraints.

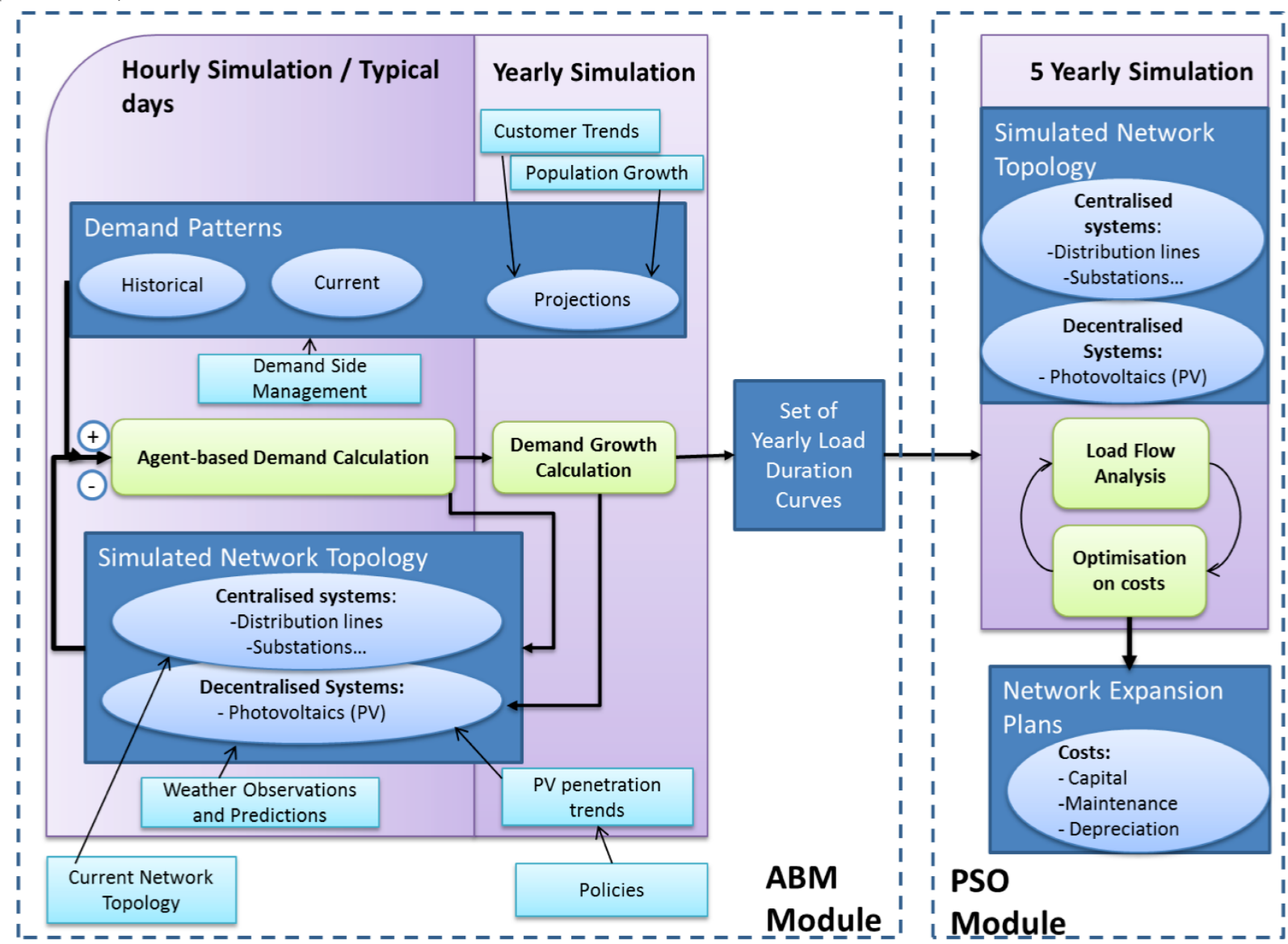

Figure 1: Schematic representation of the ABM and PSO modules and their relationships to one another. The left panel represents the short-term management module for which ABM is used. The right panel contains the long-term planning module which uses PSO. The ABM module performs simulations over 2 timeframes (hourly and yearly) using data describing the network topology and demand patterns. The output of a yearly simulation in the ABM module consists of a set of yearly load duration curves, which become input to the PSO module. The optimization module outputs network expansion plans for which costs have been minimized.

The two modeling techniques (ABM and PSO) are used distinctly over the two modules and each addresses different requirements of the analysis cycle; they can also be run independently. However, by combining them, this framework integrates different elements of analytical complexity to represent the distribution system in a realistic manner, using each method's strength.

\section{IMPLEMENTATION OF THE FRAMEWORK}

The framework described above was then implemented. The following aspects were considered that refined the way of its implementation:

- The data: data about the network assets and the load patterns were made available by the distribution company.

- The modeling approaches: as mentioned above, agent-based modeling and particle swarm optimization were selected as the techniques employed to solve the problem, which use was tailored to the available data and the need of the problem. 
- The toolkits and development environment: these were selected according to the requirements set by the problem definition, the modeling approaches selected and the data available.

Details of these three aspects are given in this section. The implementation of the platform is finally illustrated, using a case study of Townsville, Australia, for which the output of a simulation is presented, and the results are discussed.

\subsection{Description of the Data Used within the Framework}

Different data sources were made available for this project by Ergon Energy, the distribution company who partnered on this project. The two main datasets contained information about:

- The network connectivity. Information about the different assets, their characteristics and their configuration within the network were available.

- The electricity demand at different points within the network. Three types of demand data were available:

- Quarterly billing consumption data for every premise in Townsville for year 2010

- Half-hourly consumption data for a limited number of premises for year 2010

- Half-hourly load data at feeder level for year 2010

Due to the sensitivity of the information (electricity demand data), the origin of the consumption data was not identifiable; the premise identifiers were removed and only limited information about the premises was available. Such anonymity of the data required finding a way to use it that was still meaningful and as close to reality as possible. This is done as follows: 1) for each substation, a demand profile is selected randomly based on the real billing data and the type of each premise. 2) Each demand profile is then scaled to match the billing data so the average load at each substation is correct, but the shape of the load might change, depending on the selected profiles. Through this approach, the power offered by the simulation of agents at a fine level of detail is somehow lost; however, the general patterns of residential versus commercial consumption are preserved.

Table 1 gives an overview of the data types, the number of records and their use in the framework. The data was used differently at the different stages of the simulation. The ABM component uses most of the data described below, either to calibrate the model or to validate it. PSO uses some of the data as is and some after being output by the ABM simulation.

Additional datasets were also outsourced:

- PV output curves: example PV generation curves based on typical PV sizes, angles, and efficiency ratings, using TMY (typical meteorological year) data were generated using the PVWatts tool (National Renewable Energy Laboratory 2012) to be used by the PV agents.

- Historical Weather Data: this was purchased from the Bureau of Meteorology, and includes half-hourly rain, temperature, humidity and wind observations (1995-2011), plus 3 hourly cloud observations and daily global solar exposure (1990-2011). This data is used to develop more accurate models of PV output, as well as to generate future consumption profiles based on the correlation that exists between demand and the different weather parameters.

\subsection{Overview of the Modeling Approaches}

As shown in Table 1, most of the data is used by both the ABM and the PSO module; however, their use differs according to the need of the modeling technique.

In the ABM module, each asset that is described in the connectivity data is implemented as an agent. Its characteristics are set, as well as its connection to other agents according to the network hierarchy description. For each agent, a demand agent is also created and referenced so that at each time step of the simulation, the electricity consumed by it (in the case of premises e.g.) or flowing through it (for feeders, e.g.) can be calculated. In the case of generator agents, a supply agent is linked and the electricity produced at each time step is given according to the PV output curves. Following, each simulation run of the model generates $1 / 2$-hourly demand estimates for every premise and every asset in the distribution net- 
work, starting from January 2010 and continuing for a given number of years. Each simulation run can output the following kinds of data, for any collection of network assets:

- A chronological demand curve that covers the entire simulation period.

- An average daily or weekly demand curve for each year (or each season).

- A peak day curve, or peak week curve, for each year (or for each season).

These types of outputs are then transformed to be used by the PSO module. The $1 / 2$ hourly demand for the peak day for each asset is passed as a matrix to the MATLAB program along with the data associated with distribution and zone substations, distribution feeders, PVs and batteries as well as the required data for converting the line loss and reliability indices to genuine dollar. The PSO outputs a vector of solutions that indicates the mix of network upgrades and installation of DGs that minimize the cost of the network as well as the associated total cost of the network upgrade.

Table 1 : Data types used in the framework, and description of their use.

\begin{tabular}{|c|c|c|c|c|}
\hline \multicolumn{2}{|r|}{ Data Type } & Items & $\begin{array}{c}\text { Number of } \\
\text { items in models }\end{array}$ & Use in Framework \\
\hline \multirow{5}{*}{\multicolumn{2}{|c|}{$\begin{array}{l}\text { Network configuration } \\
\text { (92,261 asset agents) }\end{array}$}} & $\begin{array}{l}\text { Overhead Power Lines } \\
(845 \mathrm{~km})\end{array}$ & 5,073 & $\begin{array}{ll}\text { ABM } & \text { (Calibration) } \\
+ \text { PSO } & \end{array}$ \\
\hline & & $\begin{array}{l}\text { Underground power } \\
\text { cables }(335 \mathrm{~km})\end{array}$ & 1,468 & $\begin{array}{l}\text { ABM (Calibration) } \\
+ \text { PSO }\end{array}$ \\
\hline & & Transformers & 2,334 & $\begin{array}{l}\text { ABM (Calibration) } \\
+ \text { PSO }\end{array}$ \\
\hline & & $\begin{array}{l}\text { Switches/Isolating De- } \\
\text { vices }\end{array}$ & 3,945 & $\begin{array}{l}\text { ABM (Calibration) } \\
+\mathrm{PSO}\end{array}$ \\
\hline & & Premises & 79,441 & $\begin{array}{l}\text { ABM (Calibration) } \\
+ \text { PSO }\end{array}$ \\
\hline \multirow{7}{*}{$\begin{array}{l}\text { Electricity } \\
\text { load de- } \\
\text { mand }\end{array}$} & \multirow[t]{3}{*}{$\begin{array}{l}\text { Billing Data } \\
(79,441 \text { premises })\end{array}$} & Commercial & 5,820 & $\begin{array}{l}\text { ABM (Calibration) } \\
+ \text { PSO (transformed) }\end{array}$ \\
\hline & & Residential & 72,471 & $\begin{array}{l}\text { ABM (Calibration) } \\
+ \text { PSO (transformed) }\end{array}$ \\
\hline & & Industrial & 1,150 & $\begin{array}{l}\text { ABM (Calibration) } \\
+ \text { PSO (transformed) }\end{array}$ \\
\hline & \multirow{3}{*}{$\begin{array}{l}1 / 2 \text { hourly Metered } \\
\text { Data at premises } \\
(385 \text { premises })\end{array}$} & Commercial & 230 & ABM (Calibration) \\
\hline & & Residential & 87 & ABM (Calibration) \\
\hline & & Industrial & 67 & ABM (Calibration) \\
\hline & $\begin{array}{l}1 / 2 \text { hourly Metered } \\
\text { data at feeder level }\end{array}$ & & 90 & ABM (Validation) \\
\hline
\end{tabular}

\subsection{Choice of the Toolkits for Implementation of the Framework}

The framework presented above is implemented using Eclipse RCP (Rich Client Platform) (McAffer and Lemieux 2006).

It has MASON (Luke et al. 2005) as its agent-based modeling simulation engine, allowing the platform to be flexible and extensible through the use of Eclipse plugins. A wide range of ABM toolkits were investigated for their suitability to this application, based on the literature ((Nikolai and Madey 2009; Berryman 2008; Jackson et al. 2006; Najlis et al. 2001) and later on narrowed down according to a set of criteria specific to this project. These requirements specified that the toolkit should: have an open-source license type, use JAVA due to previous experience in software development, be fast in execution and applicable to large number of agents, have qualities of self-organization, adaptation, and causality in networks as defined by (Berryman 2008), be modular and extensible and be implementable on the Eclipse 
platform. MASON answered these criteria and was therefore selected and integrated into the Eclipse platform. While MASON provides many functionalities through its GUI, such as the upload of models, visualization of the agents movement and graphs of the outputs, these were decoupled and Eclipse RCP was used for the GUI. Only the simulation engine that contains the methods relating to the scheduling of the agents and those allowing stepping through time were kept for the simulation part.

The particle swarm optimization module is implemented in MATLAB because of its user-friendly programming language designed for numerical computation. It also has several capabilities, such as ease in implementing algorithms and visualizing outputs through the use of its graphing capabilities. It can also interface with many other languages such as $\mathrm{C}$ and Java, as well as Excel. This was important to allow the communication with the ABM module as currently this is done using *.csv files generated by the ABM module. Tighter integration of the two modules is a task soon to be implemented. For example, the scenario output by the optimization module will be validated by the ABM, to ensure that the proposed installation of DGs does shave the peak load as predicted.

\subsection{Demonstration of Implemented Framework}

Having defined the data used in the model, the modeling techniques and the toolkits, the implementation of the platform was possible. As stated above, the framework was implemented using Eclipse RCP and an example is given in Figure 2 which shows a part of the distribution network for Townsville, Australia.

The ABM GUI contains many panels which offer different ways of interacting with the model. When opening a model that has been created using the input data described in Section 5.1, a tree representation of the network is displayed in the central panel. The different assets composing the network are represented as nodes within a tree, where the edges represent the connection between the elements. Each asset, which node can be selected in the tree view, can have its individual characteristics inspected in the panel on the right side of the screen. Its demand is also displayed as a graph as the simulation progresses, see the right side panels. Any node within the tree can be inspected and its simulation output saved in a file that can be further analyzed at the end of the simulation run. This allows finding out those areas in the network that could potentially be at risk of reaching capacity.

The model displayed in Figure 2 was created using the data presented in Table 1. Therefore 92,261 asset agents were implemented and the corresponding demand either being consumed (for premises) or flowing through (for feeders e.g.) was associated to these agents. Scenarios were also used with assigning different penetration rates of solar panels which modified the demand for the grid differently over days as well as seasons, using the PV output curves as defined in Section 5.1.

Validation of the model was also performed, by comparing the simulated chronological demand curve aggregated at the feeder level with actual measurements for the same feeders which data were provided by Ergon Energy. Figure 3 shows the simulated and actual value for one feeder over the month of January 2010. As can be seen, the degree of accuracy varies over the month. The general shape within the days is respected, and some of the days match very closely; also the weekend consumption is lower than the ones during the week. However some peak days for this month have not all been captured which could be due to problems in the definition of the network configuration, where some premises are connected to a feeder other than the one specified in the network connectivity file. Validation of the model is still on-going and as it is being worked on, the model accuracy is gradually improving. 


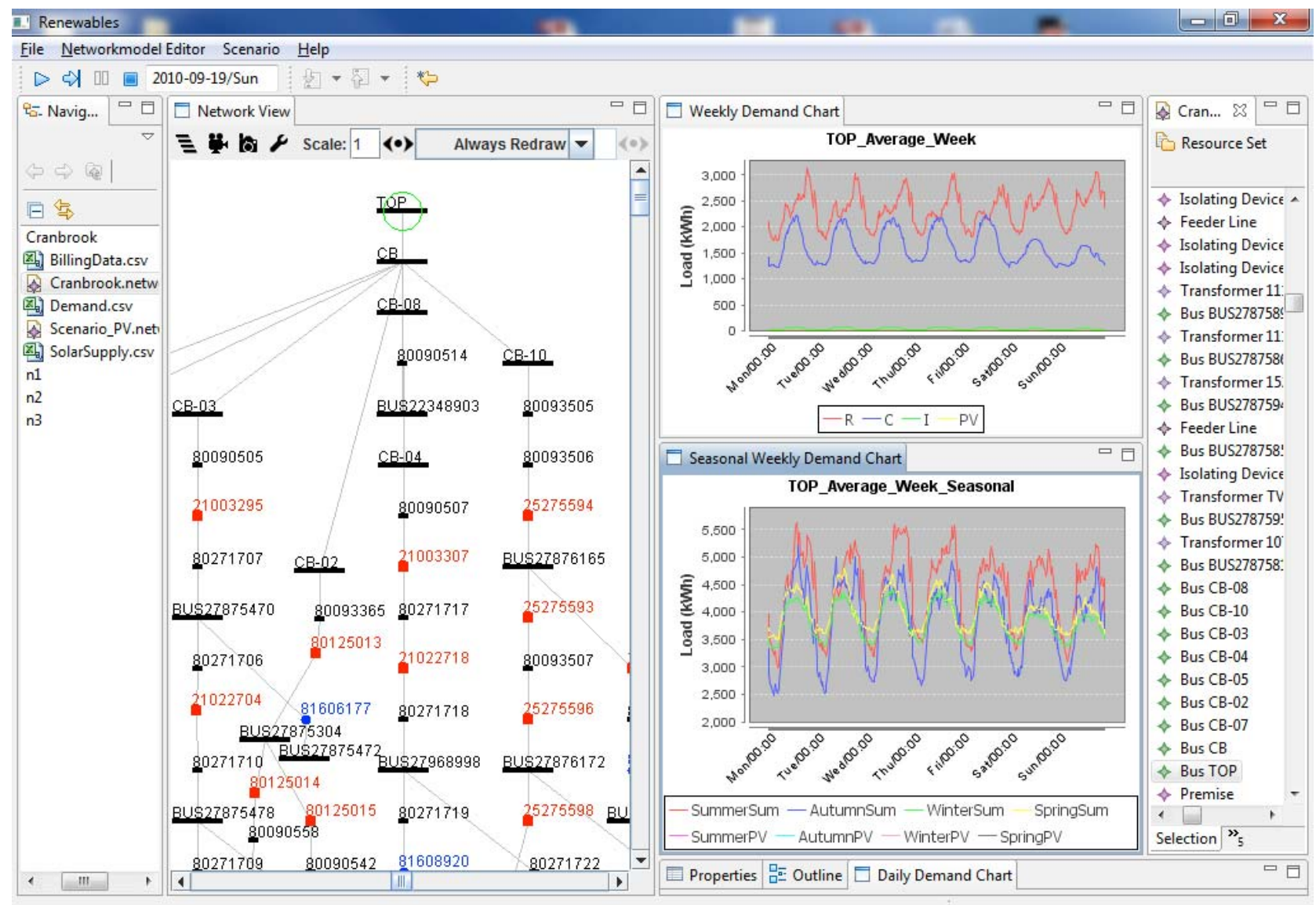

Figure 2 : Framework overview - ABM module.

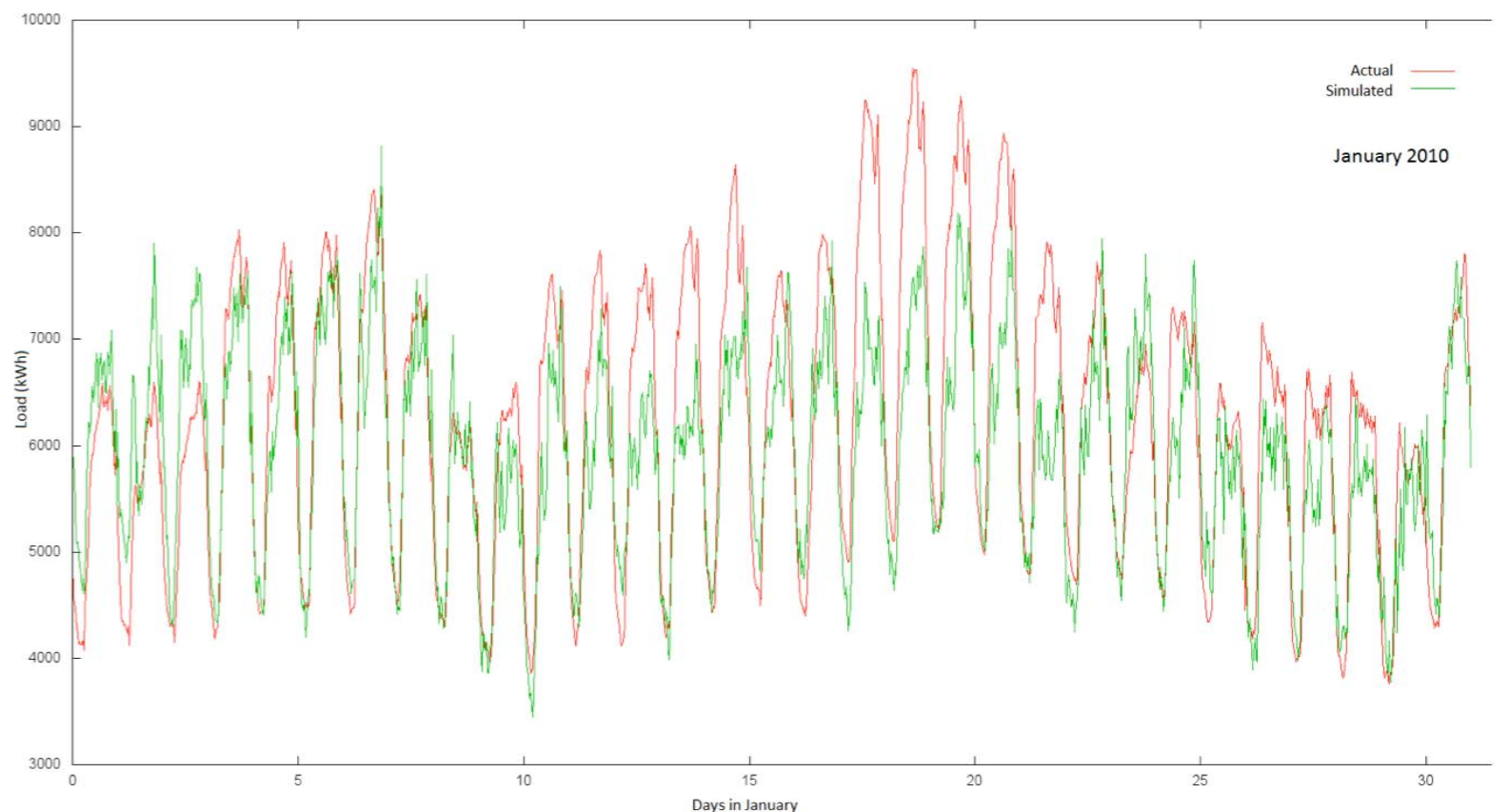

Figure 3 : Model validation - Comparison of load data measured at a given feeder and simulation output. 
From the simulations, peak consumption days can be identified, which become input to the PSO module in the form of load duration curves. Given this, an objective function is formed which is composed of the network upgrade investment cost (required cost for upgrading substations, feeders, and employed DGs), network reliability cost, and line loss cost. The application of the PSO to the given data provides a vector of particles that gives the optimal placement and sizing of DGs along with the rating of substations and feeders at different years for which the objective function (total cost) is minimized. Several preliminary optimizations have been done for the whole of Queensland, showing that optimal levels of PV can give a cost saving of $1 \%$, battery of $4.1 \%$, while PV plus battery gives a cost saving of $5.5 \%$ (Ledwich et al. 2012). Recently, the PSO has been more tightly integrated with ABM so this analysis is now being broken down to finer levels such as individual distribution substations.

In terms of performance, the ABM module can currently run 1.1 million agents per second on Intel 17-2820QM CPU; this means that if the simulation contains 1000 agents, 1100 steps will be performed in a second. It is harder however to estimate the time for the PSO module due to the variation in the parameters for each run. However, for a sample 20-year distribution network planning in presence of DGs and capacitors for a 18-bus test system, it currently takes about 1 hour on a desktop computer with a Core 2 Duo CPU E8600 at $3.33 \mathrm{GHz}$ and 3.46 GB of RAM.

\section{CONCLUSION}

Planning for the most economical and sustainable distribution network with integrating renewable generators is both a challenge and an opportunity for network managers and planners. This multi-level problem (varying temporal and geographical scales) can be answered through the combination of two modeling techniques, agent-based modeling and particle swarm optimization, which takes advantage of each method's strength over given time frames. Their integration in a streamlined manner through the development of a software platform allows both visualizing the dynamic evolution of the system on a fine temporal scale and planning for the least cost placement of renewable generators.

While the two modeling techniques have been successfully integrated into a framework, calibration of the models and their validation through the exploitation of the available data is still on-going. Also, research into modeling techniques for flexible and extensible models using a modular approach is currently undertaken, that will allow extension of the platform capability to other areas of interest as the scope of the project expands. Finally, scenarios will be built to further explore possible futures with the variation of some input parameters reflecting the consequences due to changes for example in policies, population growth in some areas or the impact that demand management measures will have on the premises consumption patterns. Development of such scenarios is key to decision-makers whose better insight into possible futures can help implement more sustainable infrastructure.

\section{ACKNOWLEDGMENTS}

The authors gratefully acknowledge the funding through the NIRAP grant, which is making this research possible, the contributions of diverse partners on this project and especially Ergon Energy for providing the data used in the framework.

\section{REFERENCES}

Australian Government. (2011). "Renewable Energy Target." Retrieved 23/08/2011, 2011, from http://www.climatechange.gov.au/government/initiatives/renewable-target.aspx.

Australian Government. (2012). "The Small-scale Renewable Energy Scheme (SRES)." Retrieved 02/04/2012, 2012, from http://ret.cleanenergyregulator.gov.au/About-the-Schemes/Small-scaleRenewable-Energy-Scheme--SRES-/about-sres.

Berryman, M. (2008). "Review of Software Platforms for Agent Based Models". D. S. T. O. Department of Defense. 
Chassin, D. P., K. Schneider and C. Gerkensmeyer (2008). "GridLAB-D: An open-source power systems modeling and simulation environment". Transmission and Distribution Conference and Exposition, 2008: 1-5.

Connolly, D., H. Lund, B. V. Mathiesen and M. Leahy (2010). "A review of computer tools for analysing the integration of renewable energy into various energy systems." Applied Energy 87(4): 10591082.

Del Valle, Y., G. K. Venayagamoorthy, S. Mohagheghi, J. C. Hernandez and R. G. Harley (2008). "Particle Swarm Optimization: Basic Concepts, Variants and Applications in Power Systems." IEEE Transactions On Evolutionary Computation 12(2): 171-195.

Ergon Energy. (2010). "Network Management Plan Part B: Electricity Supply for Regional Queensland 2010-11 to 2014-15."

Foley, A. M., B. P. Ó Gallachóir, J. Hur, R. Baldick and E. J. Mckeogh (2010). "A strategic review of electricity systems models." Energy 35(12): 4522-4530.

Hall, P. (2011). "Queensland State Government admits electricity grid failing to cope with solar power systems". Courier Mail. Brisbane.

Jackson, S. K., S. F. Railsback and S. L. Lytinen (2006). "Agent-based Simulation Platforms: Review and Development Recommendations." Simulation 82(9): 609-623.

Kirby, B. and M. Milligan (2008). "Facilitating Wind Development: The Importance of Electric Industry Structure". National Renewable Energy Laboratory.

Komor, P. (2009). "Wind and Solar Electricity: Challenges and Opportunities". PEW Center on Global Climate Change. University of Colorado.

Ledwich, G., R. Drogemuller, M. Utting, I. Ziari, F. Boulaire and A. Abeygunawardana (2012). "Planning Future Energy Grids : Renewables - Project Milestone Report". Queensland University of Technology: 1-36.

Luke, S., C. Cioffi-Revilla, L. Panait, K. Sullivan and G. Balan (2005). "MASON: A Multi-Agent Simulation Environment." Simulation: Transactions of the society for Modeling and Simulation International 82(7): 517-527.

Macal, C. M. and M. J. North (2005). "Agent-Based Modeling And Simulation". 2005 Winter Simulation Conference.

Macal, C. M. and M. J. North (2006). "Tutorial On Agent-Based Modeling And Simulation Part 2: How To Model With Agents". Winter Simulation Conference, Monterey, California, USA.

Mcaffer, J. and J.-M. Lemieux (2006). "Eclipse Rich Client Platform: designing, coding, and packaging Java applications". Upper Saddle River, NJ, Addison-Wesley.

Najlis, R., M. A. Janssen and D. C. Parker (2001). "Software Tools and Communication Issues". Proceedings of a Special Workshop on Land-Use/Land-Cover Change, Irvine, California.

National Renewable Energy Laboratory. (2012). "PVWatts - A Performance Calculator for GridConnected PV Systems." Retrieved 05/01/2012, 2012, from http://rredc.nrel.gov/solar/calculators/PVWATTS/version1/version1_index.html.

Nikolai, C. and G. Madey (2009). "Tools of the Trade: A Survey of Various Agent Based Modeling Platforms." Journal of Artificial Societies and Social Simulation 12(2): 2.

Powertech Labs Inc. (2012). "DSATools - Dynamic Security Assessment Software." Retrieved 23/05/2012, 2012, from http://www.dsatools.com/.

Queensland Government (2009). "Securing Queensland's Energy Future: Regulation for Electricity Demand Management". Queensland Government - Office of Climate Change.

Queensland Government. (2011). "Solar Bonus Scheme." Retrieved 02/04/2012, 2012, from http://ret.cleanenergyregulator.gov.au/About-the-Schemes/Small-scale-Renewable-EnergyScheme--SRES-/about-sres.

Roam Consulting. (2012). "2-4-C Lite." From http://www.roamconsulting.com.au/24CLite/index24C.php.

Schutte, S., S. Scherfke and M. Sonnenschein (2012). "mosaik - Smart Grid Simulation API". SmartGreens 2012, SciTePress. 
Wikipedia. (2011, 9 August 2011). "Agent-based model." Retrieved 23/08/2011, 2011, from http://en.wikipedia.org/wiki/Agent-based model.

Ziari, I., G. Ledwich, A. Ghosh, D. Cornforth and M. Wishart (2010). "Optimal allocation and sizing of capacitors to minimize the transmission line loss and to improve the voltage profile." Computers and Mathematics with Applications 60(4): 1003-1013.

\section{AUTHOR BIOGRAPHIES}

FANNY BOULAIRE is a Ph.D. student in the Complex Urban System Design group, QUT. She is currently applying agent-based simulation techniques to the modeling of electrical power distribution networks. Prior to this, she worked at CSIRO (2003 - 2011) developing and implementing models for diverse applications related to the urban environment. Her email address is fanny.boulaire@qut.edu.au.

Dr MARK UTTING works for QUT, developing agent-based models of future electricity grids, and is also an Associate Professor in Computer Science at the University of Waikato. From 2009-2011, he also worked for Netvalue.net.nz, using agile techniques to develop Next Generation Genomics Software. He is the author of the book 'Practical Model-Based Testing: A Tools Approach', as well as more than 50 publications on model-based testing, formal methods for object-oriented and real-time software and language design for parallelism. His email address is mark.utting@qut.edu.au.

ROBIN DROGEMULLER is the Professor of Digital Design at QUT. He holds qualifications in Architecture and Maths \& Computing. He has been working on energy implications of buildings for many years, having contributed to key work leading to the addition of energy performance requirements in the Australian building code. His major research interest is in the integration of computer based analysis tools with architectural and engineering design processes. His email address is robin.drogemuller@qut.edu.au.

GERARD LEDWICH (M'73-SM'92) received the Ph.D. degree in electrical engineering from the University of Newcastle, Australia, in 1976. He has been Chair Professor in Power Engineering at QUT, Brisbane, Australia, since 2000. His interests are in the areas of power systems, power electronics, and controls. Dr. Ledwich is a Fellow of I.E.Aust. His email address is g.ledwich@qut.edu.au.

IMAN ZIARI received his Ph.D. in Electrical Engineering from Queensland University of Technology, Australia, in 2011. He is currently working as a research fellow in Power Engineering at QUT. His interests are in the areas of distribution network planning, renewable energy resources, optimization and power quality. His email address is i.ziari@qut.edu.au. 